\title{
Pareto Set and EMOA Behavior for Simple Multimodal Multiobjective Functions
}

\author{
Mike Preuss, Boris Naujoks, and Günter Rudolph \\ Universität Dortmund, Lehrstuhl für Algorithm Engineering \\ 44221 Dortmund, Germany \\ \{mike.preuss, boris.naujoks, guenter.rudolph\}@uni-dortmund. de, \\ http://ls11-www.cs.uni-dortmund.de
}

\begin{abstract}
Recent research on evolutionary multiobjective optimization has mainly focused on Pareto-fronts. However, we state that proper behavior of the utilized algorithms in decision/search space is necessary for obtaining good results if multimodal objective functions are concerned. Therefore, it makes sense to observe the development of Pareto-sets as well. We do so on a simple, configurable problem, and detect interesting interactions between induced changes to the Pareto-set and the ability of three optimization algorithms to keep track of Pareto-fronts.
\end{abstract}

\section{Introduction}

In recent years, evolutionary multiobjective optimization (EMO) [1,2] has developed from a marginal into one of the most actively pursued areas within evolutionary computation (EC). Many new algorithms and measures have been suggested, and, with them, concepts like Pareto set and Pareto front have entered the common EC vocabulary [3]. Increasing interest in multiobjective techniques has even evoked new theoretical approaches that employ multiple objectives to simplify an originally singleobjective problem [4]. However, most of the current EMO research concentrates on processes observed in the objective space, which consists of the possibly obtainable value combinations of the considered objective functions. Undoubtedly, approximating the Paretofront well is the final aim of EMO algorithms (EMOAs), and the Pareto-set distribution may be of minor interest for estimating their performances. Nevertheless, for improving these algorithms, as well as for attaining guidelines on which of the solutions contained in the approximated Pareto-set shall eventually be implemented in a real-world situation, a well-founded understanding of Pareto-set distributions is supposed to be a major advantage.

Research on singleobjective algorithms largely focuses on population behavior in the decision space, or simplified models thereof, e.g. using basins of attraction as means of abstraction [5]. Especially for multimodal problems, numerous techniques have been invented to prevent the populations from converging to a single point too soon. Some of these, as crowding [6], are also applied in EMOAs. But diversity maintenance is only sought in objective space, to ensure good coverage of the Pareto-front. However, for at least one multimodal objective function, it is clear that this coverage cannot normally be achieved when the whole population is clustered around one local minimum of this function. We thus conjecture that a) there are situations-and these are 
not uncommon - where the Pareto-set does not share the aspired nice properties of the received Pareto-front the user normally focusses the attention on, and b) that diversity maintenance is not only needed in objective but also in decision space for successfully treating multiobjective optimization problems (MOPs): The product designer is mainly interested in a thorough covering of the Pareto-front for maximum wide scope in selecting solutions according to the (conjectured) customers' desires. This is the situation which contemporary EMOAs are designed for. But the product engineer is mainly interested in a thorough covering of the Pareto-set since it is important to know if a certain design can be realized by different parameters of the production process: Solutions may differ in sensitivity or in shorter tooling times and the like. Evidently, contemporary EMOAs are not geared toward product engineers yet.

These both sides of one medal (Pareto-front in the objective space, Pareto-set in the decision space) and the conjunction between them has not been studied in detail before. Only few theoretical results for special classes of search spaces and multiobjective functions were presented before, cf. Ehrgott [7]. But the handled cases are restricted in a way that no generalization can be foreseen. Some effort has been made in the development of test functions not only with regard to a nice behaving Pareto-front, but also with aspired properties in the decision space, cf. Okabe et al. [8]. Zhou et al. [9] propose a specialized EA to implicitly handle and profit from regularities in the objective as well as in the decision space. Such regularities stem from the test functions proposed by Okabe et al. [8] and can not be expected generally.

\section{Aims and Methods}

Our approach is constructive; on a minimalist bimodal bicriteria test problem, we study structural changes of true Pareto-set and Pareto-front on a set of targeted modifications. These are derived both analytically and empirically, the latter employing grid-based and stochastic enumerators. Furthermore, we observe how different EMOAs cope with the original problem and the changes. More detailed, we try to answer the following questions:

- How do Pareto-set distributions change when the problem is modified? Are there unexpected outcomes?

- Are different EMOAs able to cover Pareto-set and Pareto-front well?

- Are there consistent similarities/dissimilarities in the behavior of different EMOAs due to problem modifications that may highlight distinct capabilities of these?

\section{A Simple Test Problem: TWO-ON-ONE}

To deepen the insight in behavior and structure of Pareto-sets mapping onto Paretofronts, we define a very simple test problem. It consists of a polynomial function $f_{1}$ of degree four with two optima, and the sphere function $f_{2}$, which is of degree two:

$$
\begin{aligned}
f=\left(f_{1}, f_{2}\right): \mathbb{R}^{2} \rightarrow \mathbb{R}^{2}: \quad & f_{1}\left(x_{1}, x_{2}\right)=x_{1}^{4}+x_{2}^{4}-x_{1}^{2}+x_{2}^{2}-c x_{1} x_{2}+d x_{1}+20, \\
& f_{2}\left(x_{1}, x_{2}\right)=\left(x_{1}-k\right)^{2}+\left(x_{2}-l\right)^{2}
\end{aligned}
$$


The level (niveau) of the optima of $f_{1}$ can be adjusted smoothly via parameter $d$. With a positive parameter $d$, the optimum in the positive $x_{1}$ domain is lifted up in comparison to the optimum in the negative $x_{1}$ domain. Consequently, the former becomes a local optimum, while the latter remains a global optimum (asymmetric optima). With parameter $c=0$, both minimizers are located on the $x_{1}$ axis, but for increasing $c$, their connecting line is rotated counterclockwise, until its gradient is nearly 1 .

The function $f_{2}$ is unimodal, the location of its minimizer determined by parameters $k$ and $l$. For $k=l=0$ it is located in the origin, right between the minimizers of the bimodal function $f_{1}$. By variation of $k$ and $l$ the minimizer is moved away from the connecting line of the minimizers of $f_{1}$. Next to changing the Pareto-front, this also effects the Pareto-set.

Table 1: Parameter setting for the five cases of TWO-ON-ONE.

\begin{tabular}{cccccc}
\hline $\begin{array}{c}\text { Case } \\
\text { Parameter }\end{array}$ & 1 & 2 & 3 & 4 & 5 \\
\hline $\mathrm{c}$ & 10 & 10 & 10 & 10 & 10 \\
$\mathrm{~d}$ & 0 & 0 & 0.25 & 0.25 & 0.25 \\
$\mathrm{k}$ & 0 & 1 & 0 & 1 & 0 \\
1 & 0 & 0 & 0 & 0 & 1 \\
\hline
\end{tabular}

In order to allow for a theoretical analysis of the problem, five parameter settings have been fixed (Table 1, Figure 1). These result in different placements of the minimizers in search space, two for the symmetric case (both optima of $f_{1}$ identical), three for the asymmetric case (optima distinct). While all these settings are expected to lead to ordinary (generic) Pareto-fronts, the Pareto-sets are expected to look more complex.

The coordinates of the minimizers of $f_{1}$ can be determined analytically to

$$
\left(x_{1_{1,2}}^{*}, x_{2_{1,2}}^{*}\right)=\left( \pm \frac{1}{2}(\sqrt{101}+1)^{1 / 2}, \pm \frac{1}{20}(\sqrt{101}-1)(\sqrt{101}+1)^{1 / 2}\right)
$$

In cases one and two, both optima of $f_{1}$ are on the same level, ensured by $d=0$. In the former, the minimizer of the sphere function is located in the origin, where $f_{1}$ has a saddle point. In the latter, the optimum is moved right on the $x_{1}$ axis by one unit.

The first two situations are repeated for cases three and four, featuring a global optimum in the negative $x_{1}$ and $x_{2}$ domain, and a local optimum in the positive domain. It is expected that the Pareto-set establishes a connection between the global optimum of $f_{1}$ and the one of $f_{2}$ in these situations. This is due to the solutions in the global optimum of $f_{1}$ being mapped to the extremal part of the Pareto-front. Consequently, the solutions from the local optimum of $f_{1}$ may be lost.

The same situation is expected for case five, which is similar, except for a movement of the minimizer of $f_{2}$ towards the global minimizer of $f_{2}$, whereas in case four, it is brought nearer to the local minimizer. 

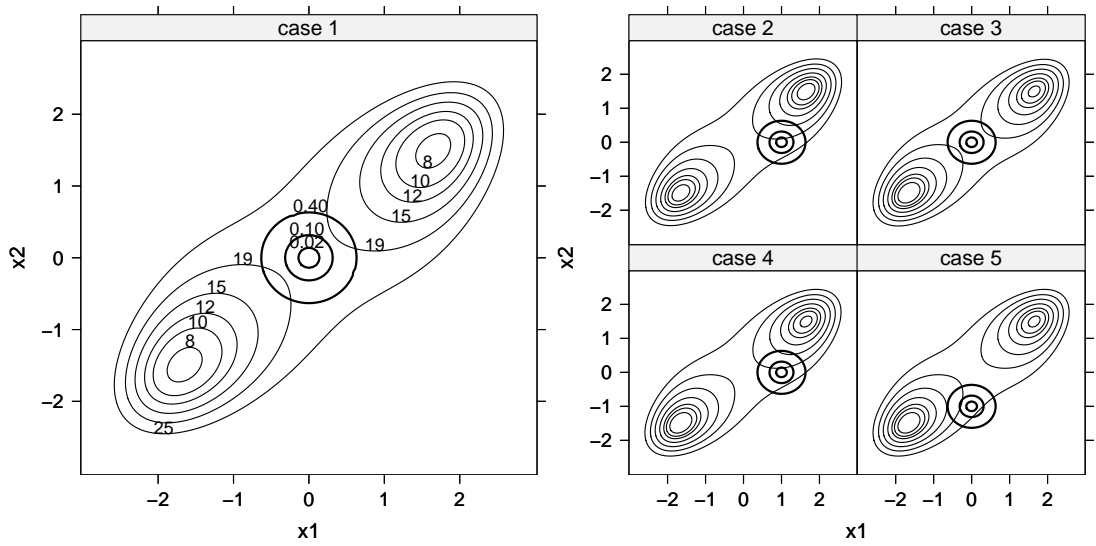

Fig. 1: Superposition of functions $f_{1}$ and $f_{2}$ (sphere) of TWO-ON-ONE for cases 1 to 5. The first two are symmetric, the last three asymmetric with the right minimizer of $f_{1}$ shifted slightly upwards and the left one downwards.

\section{Experimental Investigation of Pareto Sets}

Our expectation is that for all symmetrical cases of $f_{1}\left(f_{1}\left(\right.\right.$ optimum $\left._{1}\right)=f_{1}\left(\right.$ optimum $\left.\left._{2}\right)\right)$, the Pareto sets consist of two curves, connecting either peak with the minimizer of the sphere function $f_{2}$. For the asymmetric cases, it seems reasonable that Pareto-sets shall only contain points on a curve between the global minimizers of $f_{1}$ and $f_{2}$. Note that this expectation stems from thought experiments rather than empirical or analytical facts.

We employ two simple tools, a grid based and a stochastic enumerator, for obtaining a first, rough impression of structure and location of the Pareto-sets. Either one samples points from a given interval and keeps a list of the Pareto-optimal solutions found. As we shall see, it sometimes makes sense to use both, as the obtained results can subtly differ.

Experiment 1: Determine Pareto sets and fronts of TWO-ON-ONE.

Pre-experimental planning: First experiments were performed with a grid-based enumerator only. They revealed strange artifacts, making the Pareto-set look like consisting of vertical bars (Figure 2, left). We therefore additionally sampled by means of a stochastic enumerator.

Task: Find location of the Pareto-sets, detect deviations from the expected.

Setup: For each of the 5 cases specified in Table 1, we sample points in the interval $x_{1}, x_{2} \in[-3,3]$. The grid-based enumeration consists of $300 \times 300=90,000$ points each, the stochastic enumeration of 500,000 points each. The difference is intended as we hope for a better resolution with the latter method, to shed light on the bar-shaped artifacts. All non-dominated points are archived. 

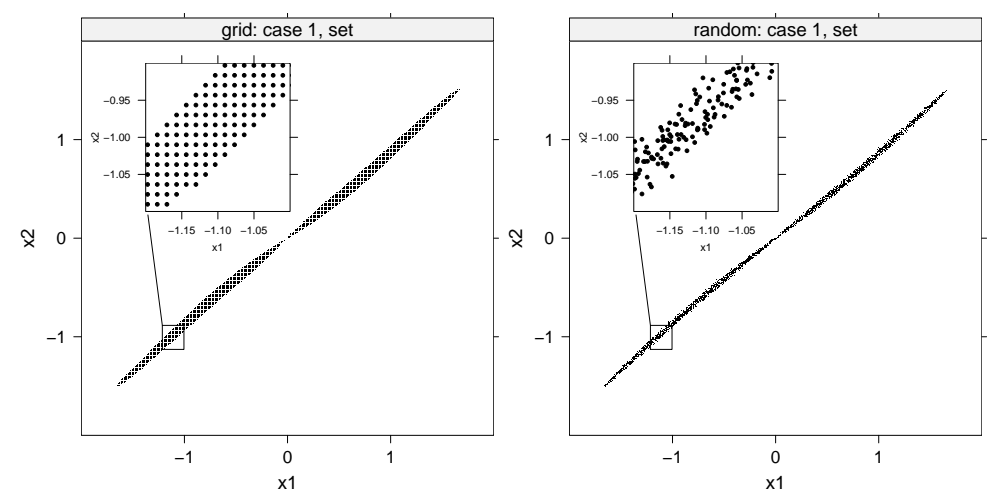

Fig. 2: Pareto-sets of case 1, obtained with grid and stochastic enumerator.

Experimentation/Visualization: Figures 2 and 3 show the most interesting of the obtained Pareto sets and fronts. All others largely comply with the previously stated expectations and are omitted due to space limitations.

Observations: The figures clearly show that neither grid nor stochastic sampling produces a clear-cut picture of the true Pareto-sets. Roughly, case 1 yields a smeared areal, propeller-like structure (Figure 2). This is surprising as we expected to see a single curve here. However, the Pareto-set appears narrower under stochastic enumeration.

For case 4 (sphere function $f_{2}$ moved towards local optimum of $f_{1}$ ), the Paretofront splits into two parts at $f_{1} \approx 8$, as visualized in Figure 3. Accordingly, the Paretoset breaks up into two distinct fragments. Note that no connection exists between the location of the sphere and the global optimum of $f_{1}$. At the left edge of its right part, the grid-based approximated Pareto-set reveals a strange curl which is not visible in the stochastically approximated Pareto-set. Pareto-fronts of cases 4 and 5 both contain pieces of very low point densities, at $17<f_{1}<19$ in the former, and $15<f_{1}<17$ in the latter case.

Discussion: We regard the obtained Pareto-set approximations for case 1 as rather misleading, and analytical investigation in $\S 5$ supports this view. However, considering the amount of sampled points ( $90 k$ and $500 k$ ), and taking into account that the latter (stochastically approximated) Pareto-set is much tighter, one may conclude even from our empirical data that the true Pareto-set indeed is most likely located on a curve and nonareal. The enumerators are probably misguided by the huge difference in gradients of $f_{1}$ and $f_{2}$ in direction of the connecting line between the two optima of $f_{1}$ and orthogonal to it. Following from that, any EMOA will experience the same situation: Practically identical values of the objective functions can have a large set of preimages and thus spread in search space.

Results obtained for case 4 show that contrary to our expectation, by far the larger Pareto-set portion resides in the range between the local optimum of $f_{1}$ and the optimum of $f_{2}$. Only where function values for $f_{1}$ are better than may be attained at the local optimum, points from the left fragment can enter the Pareto-set, resulting in a stepped Pareto-front. The curl found near $x_{1}=1$ seemingly corresponds to the low density part 

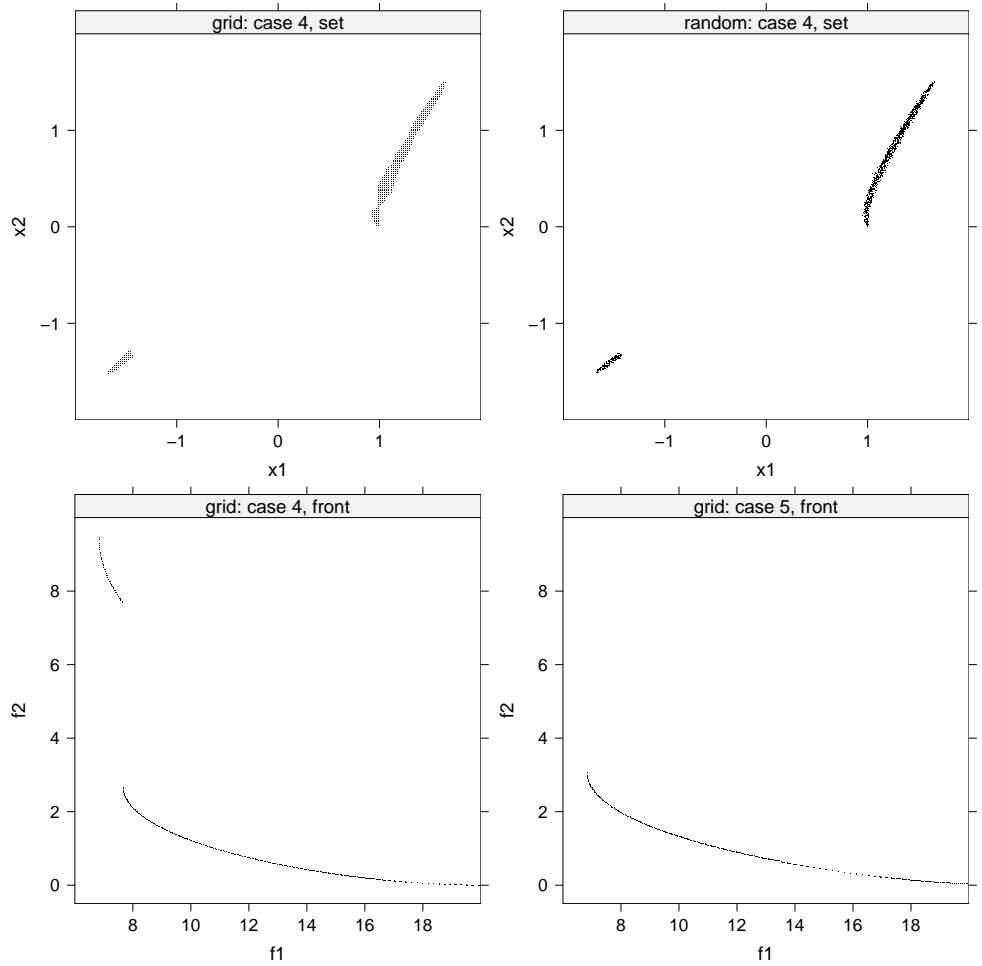

Fig. 3: Surprising Pareto sets and fronts for cases 4 and 5.

of the Pareto-front which must be located in proximity of the sphere center as values for $f_{2}$ are near 0 . The stochastic Pareto-set approximation is again tighter than the gridbased one, leading to the conjecture that the true Pareto-set is non-areal as in case 1.

Two more conclusions may be drawn from the case 4 results. Firstly, search space distances between optima of separate objective functions play a major role for the composition of Pareto-sets, and secondly, it is necessary to keep the population of EMOAs spread over several local optima of the treated objective functions during an optimization run.

\section{Analytical Derivation of Pareto Sets}

The Pareto-set for case 1 can be derived analytically but its analytic expression is too complex and space-consuming to be presented here. Instead, we suggest the linear approximation

$$
\left(x_{1}, \frac{\sqrt{101}-1}{10} x_{1}\right) \text { for } \quad x_{1} \in\left[-\frac{1}{2}(\sqrt{101}+1)^{1 / 2}, \frac{1}{2}(\sqrt{101}+1)^{1 / 2}\right]
$$


whose deviation from the true convex-concave curve is less than 0.045 for all $x_{1}$ above. In any case, the Pareto-set is a 1-dimensional connected set and not an areal set of higher dimension as the output of the grid or stochastic enumerator might suggest (see fig. 2).

As can be seen from the symmetry $f\left(x_{1}, x_{2}\right)=f\left(-x_{1},-x_{2}\right)$ the entire Paretofront can be built solely by positive (or negative) points of the Pareto-set. Thus, it may happen that an EMOA approximating the Pareto-front quite well with regard to the Smetric has found only points in the decision space with, say, positive components. As a consequence, a good value for the S-metric tells only half the story.

The Pareto-sets of the other cases are also amenable to an analytic solution but the expressions are far away from being manageable easily. This observation is quite counter-intuitive given the pretended simple expressions and structural design of the objective functions.

\section{Behavior of EMOAs on TWO-ON-ONE}

Whereas Pareto-sets and fronts of problem TWO-ON-ONE have been explored in $\S 4$ and determined analytically in $\S 5$, we now turn to the behavior of different EMOAs in a second experiment. Note that it is not intended to argue in favor of or against any algorithm here, but rather to detect possible differences.

Algorithms We invoke two standard techniques next to a new development within the field. The Pisa framework ${ }^{1}$ is used to conduct the referred optimization runs. Here, the TWO-ON-ONE problem has been implemented as a variator, which can be optimized with respect to different objectives and multiple selectors. Among the set of available selectors, NSGA-II and SPEA2 are chosen, because these appear to be the currently most well-known and commonly used algorithms in the field [1,2]. Additionally, the more recent SMS-EMOA $[10,11]$ is tested within this framework. The SMSEMOA was designed for featuring a performance measure, namely the hypervolume or S-metric, as secondary ranking criterion in a NSGA-II like manner. The additional effort for a third algorithm in the study seems to be justified, because the SMS-EMOA was found to spread solutions more nicely over Pareto-fronts than the other two algorithms. This is surely due to the different selection criterion employed. We investigate if this also holds for our simple test problem and especially if it can be recognized within the generated Pareto-sets.

Measures To detect differences in algorithm behaviors on the most interesting cases, we define two simple measures. For case 1, we measure if the resulting population $P$ is fairly distributed over the left and right wings of the Pareto-set by taking the fraction on the less crowded wing into account:

$$
\text { fair }(P)=\frac{1}{2}-\frac{\min \left(\mid\left\{\text { individual } \in P: x_{1}<0\right\}|,|\left\{\text { individual } \in P: x_{1} \geq 0\right\} \mid\right)}{|P|}
$$

\footnotetext{
${ }^{1}$ PISA - Platform and Programming Language Independent Interface for Search Algorithms, ETH Zurich, www.tik.ee.ethz.ch/pisa/
} 
For case 4, we are interested in the fraction of points in proximity of the global optimum of $f_{1}$, corresponding to the search points in the left half of the search space:

$$
\operatorname{left}(P)=\frac{\mid\left\{\text { individual } \in P: x_{1}<0\right\} \mid}{|P|}
$$
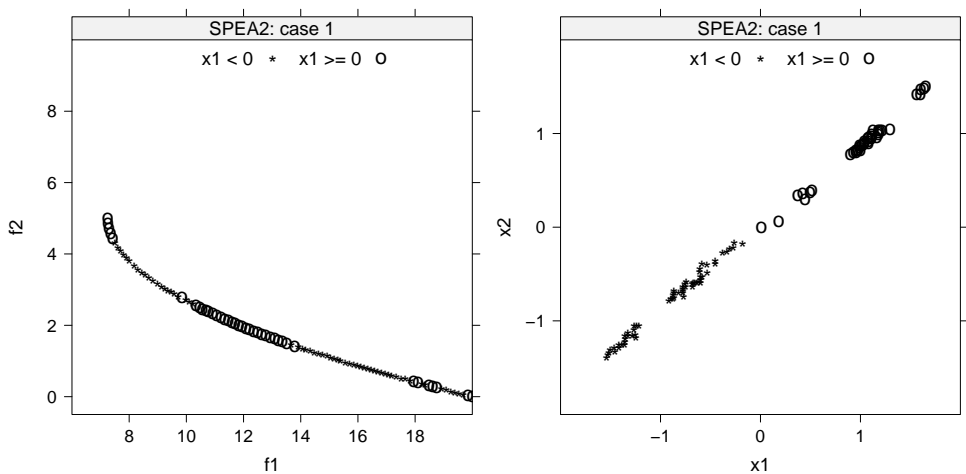

Fig. 4: Pareto-front (left) and Pareto-set (right) of a single SPEA2 run on case 1.

Experiment 2: Search Space Behavior of EMOAs on function TWO-ON-ONE.

Pre-experimental planning: First runs indicated that results on cases 2, 3, and 5 are comparable for all three algorithms. We thus focused on cases 1 and 4 . For case 1 it was found that at least 50 runs are necessary to get a detailed picture of differences in measure fair $(P)$, for case 4, 20 runs seemed sufficient.

Task: Detect differences in the obtained Pareto-sets and fronts that may be related to test problem properties. We employ bootstrap permutation tests with 49, 999 replicates and significance level $5 \%$ for the measured data.

Setup: The decision space was limited to $f_{1}, f_{2} \in[-50,50]$, thereby enclosing the region around the optima of $f_{1}$ and $f_{2}$, and a certain amount of space the algorithms have to bypass to get there. All three algorithms, NSGA-II, SPEA2, and SMS-EMOA, are run with a population size of 100 for 30,000 evaluations, otherwise utilizing default parametrizations. For case 1 and 4, 50 and 20 runs are performed, respectively.

Experimentation/Visualization: Figure 4 depicts a typical outcome for case 1. More extreme population distributions with almost all individuals on one wing of the Paretoset also happen. In figure 5, resulting Pareto-sets for two different algorithms are presented, again, typical runs are chosen. Figure 6 holds histograms for the distributions of measures fair $(P)$ on case 1 and $\operatorname{left}(P)$ on case 4 .

Observations: Figure 4 demonstrates that for symmetric optima, the Pareto-front often contains large chunks of points originating from the proximity of different minimizers. Accordingly, the approximated Pareto-sets show corresponding clouds of points, unevenly distributed over the true Pareto-set. For case 4, Figure 5 shows that the algorithms are able to spread their populations over both important parts of the Pareto-set. 

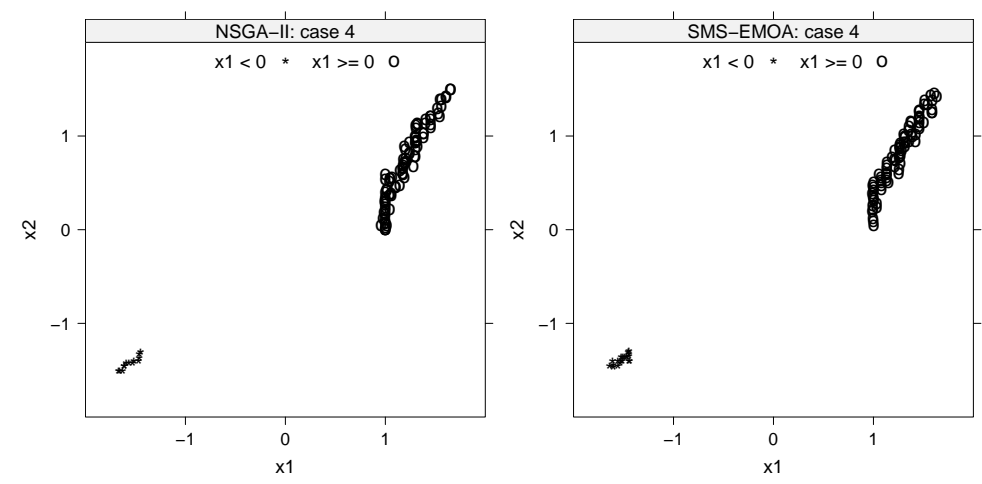

Fig. 5: Pareto-sets of single NSGA-II (left) and SMS-EMOA (right) runs on case 4.

However, the shape of the clouds near the global minimizer of $f_{1}$ is different: NSGA-II often forms lines of points in that region, whereas the SMS-EMOA rather builds areal structures.
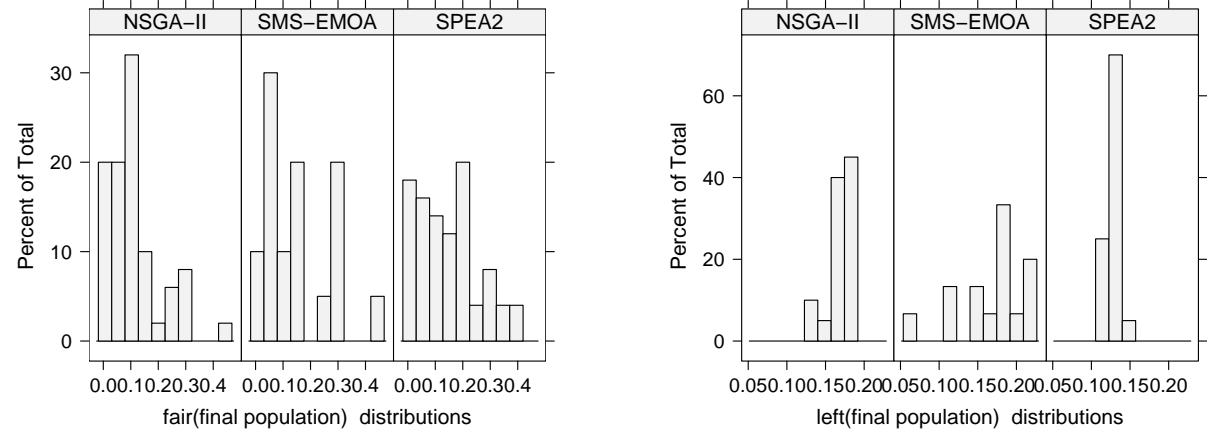

Fig. 6: Histograms for measures fair $(P)$ on case 1 and left $(P)$ on case 4.

Discussion: For case 1, hypothesis testing reveals a slight difference between NSGA-II and SMS-EMOA, p-Value 0.071, and a strong one between NSGA-II and SPEA2, pValue 0.030 . SMS-EMOA and SPEA2 may be considered behaving relatively similar, p-Value 0.427. Visual comparison of histograms depicted in Figure 6 sees the SMSEMOA as something inbetween NSGA-II and SPEA2. In effect, NSGA-II covers both wings of the Pareto-set more evenly on average, its fair() measure is 0.110 , compared to 0.149 and 0.152 for SPEA2 and SMS-EMOA, respectively.

All three algorithms cope surprisingly well with case 4 . Here, hypothesis tests hint to a similarity between SMS-EMOA and NSGA-II, p-Value 0.468 , and sharp distinction between SPEA2 and SMS-EMOA, and SPEA2 and NSGA-II, both p-Values 0.001. As indicated by the histograms, NSGA-II and SMS-EMOA both place more points near the global optimizer, their left() measures are 0.169 and 0.167 , respectively. SPEA2 only puts $13.1 \%$ of its final population there. Unfortunately, we are currently not able to explain what makes the algorithms behave differently in this respect. 


\section{Summary and Outlook}

The main message of the work presented here is our belief in the fact that a neat covering of the Pareto-front is not sufficient for meeting the needs of all clients that may use EMOAs. Therefore, future versions of EMOAs should also take into account a proper covering of the Pareto-set. Evidently, contemporary EMOAs cannot deliver this kind of behavior. For this purpose we need also an effective measure for assessing the quality of a solution set in decision space-similarly to the S-metric in objective space. And if we have such a measure then it can be integrated into the EMOA for guiding the search-similarly to the S-metric in the SMS-EOMA.

Needless to say, if we follow this avenue we have to deepen our insights into the behavior of EMOAs in the decision space. Such an analysis can be quite counter-intuitive and surprising as our seemingly simple test problem has revealed. We are convinced that a thorough analysis of the interaction between Pareto-front and Pareto-set will eventually lead to new insights, new search operators, and even better EMOAs.

\section{References}

1. Deb, K.: Multi-Objective Optimization using Evolutionary Algorithms. Wiley, Chichester, UK (2001)

2. Coello Coello, C.A., Van Veldhuizen, D.A., Lamont, G.B.: Evolutionary Algorithms for Solving Multi-Objective Problems. Kluwer Academic Publishers, New York (2002)

3. Coello, C.A.C.: Evolutionary multi-objective optimization: a historical view of the field. IEEE Computational Intelligence Magazine 1(1) (2006) 28-36

4. Neumann, F., Wegener, I.: Minimum spanning trees made easier via multi-objective optimization. In Beyer, H.G., ed.: GECCO '05: Proceedings of the 2005 conference on Genetic and evolutionary computation, New York, NY, USA, ACM Press (2005) 763-769

5. Preuss, M., Schönemann, L., Emmerich, M.: Counteracting genetic drift and disruptive recombination in $(\mu+\lambda)$-ea on multimodal fitness landscapes. In Beyer, H.G., ed.: GECCO '05: Proceedings of the 2005 conference on Genetic and evolutionary computation, New York, NY, USA, ACM Press (2005) 865-872

6. De Jong, K.A.: An analysis of the behavior of a class of genetic adaptive systems. $\mathrm{PhD}$ thesis, University of Michigan (1975)

7. Ehrgott, M.: Multicriteria Optimization. 2 edn. Springer, Berlin (2005)

8. Okabe, T., Jin, Y., Olhofer, M., Sendhoff, B.: On Test Functions for Evolutionary Multiobjective Optimization. In et al., X.Y., ed.: Parallel Problem Solving from Nature - PPSN VIII. Lecture Notes in Computer Science Vol. 3242, Springer, Berlin (2004) 792-802

9. Zhou, A., Zhang, Q., Jin, Y., Tsang, E., Okabe, T.: A model-based evolutionary algorithm for bi-objective optimization. In: Evolutionary Computation Congress (CEC'05), Edinburgh, UK. Volume 2., Piscataway NJ, IEEE Press (2005) 2568-2575

10. Emmerich, M., Beume, N., Naujoks, B.: An EMO algorithm using the hypervolume measure as selection criterion. In Coello, C.A.C., et al., eds.: Evolutionary Multi-Criterion Optimization: 3rd Int'1 Conf. (EMO 2005), Springer, Berlin (2005) 62-76

11. Naujoks, B., Beume, N., Emmerich, M.: Multi-objective optimisation using S-metric selection: Application to three-dimensional solution spaces. In: Evolutionary Computation Congress (CEC'05), Edinburgh, UK. Volume 2., Piscataway NJ, IEEE Press (2005) 12821289 